University of Nebraska - Lincoln

DigitalCommons@University of Nebraska - Lincoln

Stephen Ducharme Publications

Research Papers in Physics and Astronomy

$10-2009$

\title{
Nanoscale Domain Patterns in Ultrathin Polymer Ferroelectric Films
}

\author{
Pankaj Sharma \\ University of Nebraska-Lincoln, psharma@huskers.unl.edu \\ Timothy J. Reece \\ University of Nebraska-Lincoln, reecetj@unk.edu \\ Daniel W. Wu \\ University of Nebraska-Lincoln \\ Vladimir M. Fridkin \\ Institute of Crystallography, Moscow, fridkin@ns.crys.ras.ru \\ Stephen Ducharme \\ University of Nebraska-Lincoln, sducharme1@unl.edu \\ See next page for additional authors
}

Follow this and additional works at: https://digitalcommons.unl.edu/physicsducharme

Part of the Condensed Matter Physics Commons, and the Materials Science and Engineering Commons

Sharma, Pankaj; Reece, Timothy J.; Wu, Daniel W.; Fridkin, Vladimir M.; Ducharme, Stephen; and Gruverman, Alexei, "Nanoscale Domain Patterns in Ultrathin Polymer Ferroelectric Films" (2009). Stephen Ducharme Publications. 63.

https://digitalcommons.unl.edu/physicsducharme/63

This Article is brought to you for free and open access by the Research Papers in Physics and Astronomy at DigitalCommons@University of Nebraska - Lincoln. It has been accepted for inclusion in Stephen Ducharme Publications by an authorized administrator of DigitalCommons@University of Nebraska - Lincoln. 


\section{Authors}

Pankaj Sharma, Timothy J. Reece, Daniel W. Wu, Vladimir M. Fridkin, Stephen Ducharme, and Alexei Gruverman 


\title{
Nanoscale domain patterns in ultrathin polymer ferroelectric films
}

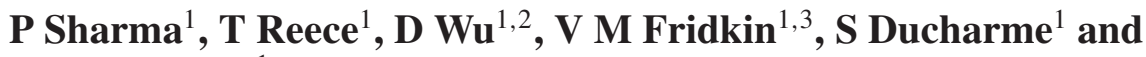 \\ A Gruverman ${ }^{1}$ \\ ${ }^{1}$ Department of Physics and Astronomy, University of Nebraska Lincoln, \\ Lincoln, NE 68588-0111, USA \\ ${ }^{2}$ Department of Physics, North Carolina State University, Raleigh, NC 27695, USA \\ ${ }^{3}$ Institute of Crystallography, Russian Academy of Sciences, Moscow 117333, Russia \\ E-mail: agruverman2@unl.edu
}

Received 14 July 2009, in final form 5 October 2009

Published 30 October 2009

Online at stacks.iop.org/JPhysCM/21/485902

\begin{abstract}
High-resolution studies of domain configurations in Langmuir-Blodgett films of ferroelectric polymer poly(vinylidene fluoride-trifluoroethylene), $\mathrm{P}(\mathrm{VDF}-\mathrm{TrFE})$, have been carried out by means of piezoresponse force microscopy (PFM). Changes in film thickness and morphology cause significant variations in polarization patterns. In continuous films and nanomesas with relatively low thickness/grain aspect ratio $(<1 / 10)$, the relationship between the average domain size and thickness follows the Kittel law. Nanomesas with high aspect ratio $(>1 / 5)$ exhibit significant deviations from this law, suggesting additional surface-energy-related mechanisms affecting the domain patterns. Polarization reversal within a single crystallite has been demonstrated and local switching parameters (coercive voltage and remnant

piezoresponse) have been measured by monitoring the local hysteresis loops. Reliable control of polarization at the sub-grain level demonstrates a possibility of studying the mechanism of the intrinsic switching behavior down to the molecular scale.
\end{abstract}

(Some figures in this article are in colour only in the electronic version)

\section{Introduction}

Reorientation of spontaneous polarization of ferroelectric materials by an external electric field opens up the possibility of electrical control of their functional properties and provides a physical basis for a number of electronic device applications, such as nonvolatile memories and mass data storage. While most fundamental studies and engineering efforts until recently have been focused on inorganic ferroelectrics, advances in materials design and synthesis of new organic solids has drawn more attention to organic ferroelectric compounds. Poly(vinylidene fluoridetrifluoroethylene), $\mathrm{P}(\mathrm{VDF}-\mathrm{TrFE})$, is the best known and most widely used ferroelectric polymer because of its relatively high polarization value and electromechanical activity $[1,2]$. In spite of a very high coercive field required for switching [3], discovery of ferroelectricity in ultrathin $\mathrm{P}(\mathrm{VDF}-\mathrm{TrFE})$ films with demonstrated electrical switchability in the range of just several volts [4] makes integration of these films into allorganic electronic systems more feasible. Recent fabrication of highly ordered arrays of $\mathrm{P}(\mathrm{VDF}-\mathrm{TrFE})$ nanomesas for nonvolatile memories [5] underscored the importance of improving crystallinity to attain spatially uniform switching properties for device applications.

In view of rapidly progressing technology scaling, investigation of static and dynamic properties of ferroelectrics at the nanoscale becomes increasingly important. Polymer ferroelectric films, where thickness can be reduced down to a single molecular layer, can be considered as the model system for addressing fundamentally important issues of critical thickness and intrinsic switching behavior. While investigation of the switching properties and polarization stability in polymer ferroelectrics have been studied extensively on the macroscopic level [6], examining the properties of nanoscale structures requires application of techniques capable of testing their static and dynamic polarization behavior with the appropriate spatial and time resolution. Currently, piezoresponse force microscopy (PFM) is the most widely used technique for nanoscale characterization of the static and dynamic polarization behavior in ferroelectrics. The 
advantage of PFM is that it allows simultaneous highresolution topographic and polarization imaging along with controlled nanoscale modification of the domain structure. Historically, due to their strong piezoelectric activity, PVDF films have been chosen as a model material system to test the viability of the piezoresponse method and to perform electromechanical imaging of poled domains [7, 8]. Recently, extensive studies of the relaxation and switching behavior in Langmuir-Blodgett $\mathrm{P}(\mathrm{VDF}-\mathrm{TrFE})$ films have been carried out by means of PFM [9, 10]. A combination of macroscopic electrical measurements and PFM switching studies has been employed to discern the mechanism of polarization switching kinetics in spun-on PVDF films [11]. However, very few reports have succeeded in unambiguous high-resolution detection of polarization [12] as the PFM signal is strongly influenced by electrostatic tip-sample contributions, by the presence of amorphous phase and complex morphology, and can additionally be affected by morphological instability as a result of electrically induced decomposition of the polymer sample. Thus, manipulation of polarization at the molecular level and addressing the scaling behavior in ferroelectric polymers is still an unsolved challenge.

In this paper, we report the results of high-resolution PFM studies of domain configurations in ultrathin LangmuirBlodgett P(VDF-TrFE) films to address the effect of film thickness and morphology on polarization patterns. Nanoscale imaging and control of polarization at the sub-grain level demonstrates a possibility of studying the intrinsic switching behavior and critical thickness effect on polarization stability.

\section{Experimental section}

Ultrathin films of ferroelectric copolymer P(VDF-TrFE) with a molar content ratio of 80:20 were fabricated on highly doped $(0.001-0.005 \Omega \mathrm{cm}) \mathrm{Si}$ substrates using a horizontal Schaefer variation of the Langmuir-Blodgett (LB) transfer technique. A Langmuir layer was dispersed on an ultrapure water subphase from a $0.05 \%$ solution of $\mathrm{P}(\mathrm{VDF}-\mathrm{TrFE})$ in dimethyl sulfoxide. Then films were deposited by touching the surface with an $\mathrm{Si}$ substrate. Deposition was carried out at a constant subphase surface pressure of $5 \mathrm{mN} \mathrm{m}^{-1}$ and temperature of $25^{\circ} \mathrm{C}$. Film thickness was controlled by the number of deposition cycles. After deposition, the films were annealed at $130^{\circ} \mathrm{C}$ for a period of $90 \mathrm{~min}$ with subsequent cooling to room temperature at a rate of $1^{\circ} \mathrm{C} \mathrm{min}-1$ in a microprocessor-controlled forced air oven. The PVDF-TrFE copolymer does not form a true single molecular layer and, as such, the average thickness of a nominal monolayer (ML), i.e. a layer obtained during a single transfer cycle, is about $1.8 \mathrm{~nm}$. The ML thickness was obtained from the cross-sectional analysis of the topographic AFM images. Further details of film preparation can be found elsewhere $[13,14]$. In the present work, a set of P(VDF-TrFE) films with thicknesses of 1, 3, 6 and 12 ML have been studied. Film morphology and grain sizes were controlled by subjecting the films to additional annealing under the same conditions as above. Detailed studies of the effect of annealing conditions on $\mathrm{P}(\mathrm{VDF}-\mathrm{TrFE})$ film morphology are reported in $[15,16]$.

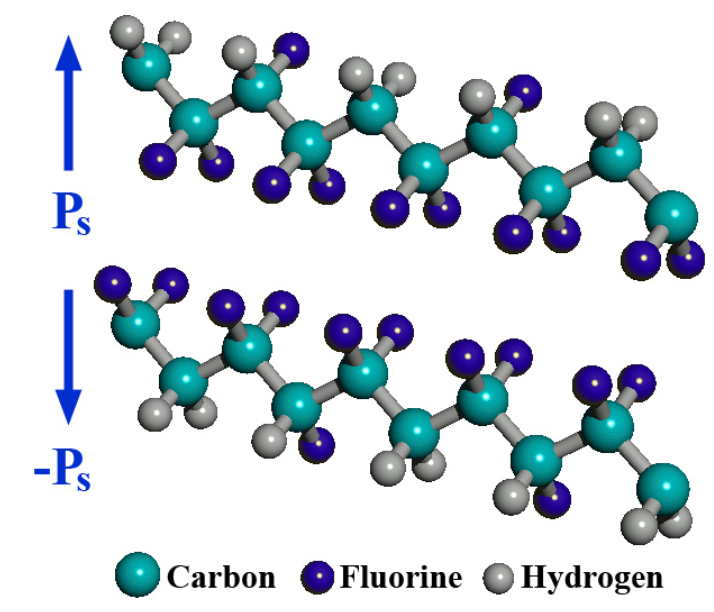

Figure 1. Ball-and-stick model of the P(VDF-TrFE) molecules with opposite directions of dipole moments.

In piezoresponse imaging of ferroelectrics, a periodic bias $V_{\text {tip }}=V_{0} \cos \omega t$ with amplitude $V_{0}$ well below the coercive voltage is applied to the probing tip in contact with the surface. Due to the converse piezoelectric effect, application of the periodic bias results in a surface displacement $z=$ $z_{0} \cos (\omega t+\varphi)$, detected by the same tip. By scanning the top surface with the voltage-modulated tip, the two-dimensional maps of piezoresponse amplitude $z_{0}$ and piezoresponse phase $\varphi$ can be generated along with the conventional topographic image. While in domains with out-of-plane polarization the local PFM amplitude signal is determined by the value of the effective piezocoefficient $d_{\text {eff }}$ and is a linear function of the modulation voltage, i.e. $z_{0}=d_{\text {eff }} V_{\text {tip }}$, the PFM phase signal changes from $0^{\circ}$ for domains with the polarization direction coinciding with the applied field to $180^{\circ}$ for domains directed in the opposite direction. For a ferroelectric sample containing only antiparallel domains with out-of-plane polarization this imaging method produces a PFM phase image consisting of dark and bright regions, while a PFM amplitude image exhibits dark lines on the bright background representing $180^{\circ}$ domain walls [17].

A commercial atomic force microscope (Asylum MFP3D) was used in this study. Domain visualization has been performed by applying a high-frequency modulating voltage (400-800 kHz, 1.0-1.5 V), using Pt-Ti-coated silicon (Mikromasch) and Au-coated SiN tips (Olympus). Local piezoelectric hysteresis loops were measured in fixed locations on the film as a function of a dc bias superimposed on ac modulation voltage.

\section{Results and discussion}

On the molecular level, ferroelectric polarization in P(VDFTrFE) arises from alignment of molecular dipoles formed by positive hydrogen ions and negative fluorine ions. Figure 1 shows the molecular structure of $\mathrm{P}(\mathrm{VDF}-\mathrm{TrFE})$ which consists of $-\mathrm{CH}_{2}-\mathrm{CF}_{2}-$ and $-\mathrm{CHF}-\mathrm{CF}_{2}-$ units rigidly attached to the main-chain carbon atoms [6]. The all-trans molecular conformation results in the dipoles oriented perpendicular to 

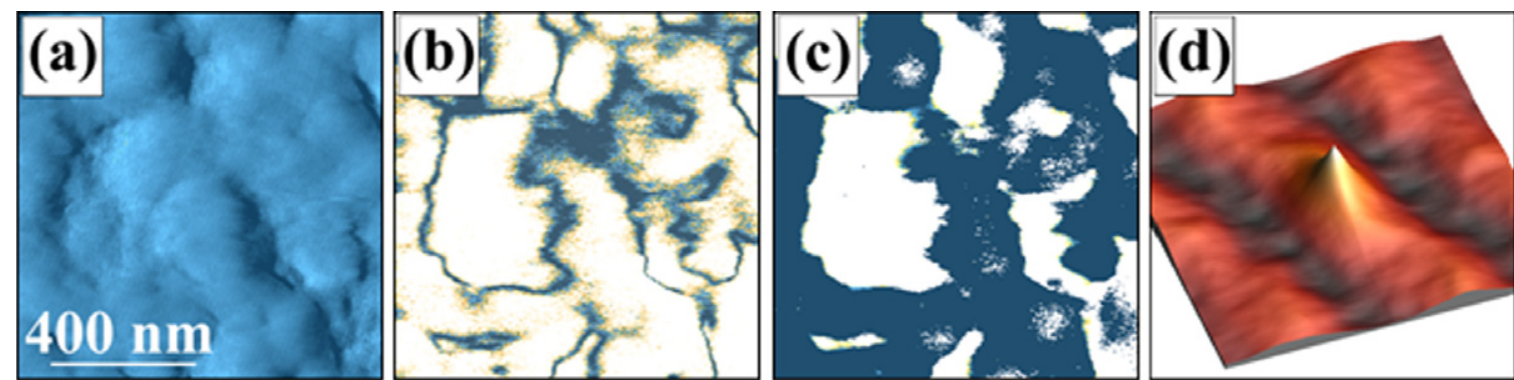

Figure 2. Domain imaging in 12 ML P(VDF-TrFE) film subjected to one cycle of annealing: (a) topography; (b) PFM amplitude; (c) PFM phase; and (d) 3D auto-correlation image of the PFM phase data (scale is arbitrary along the vertical axis).
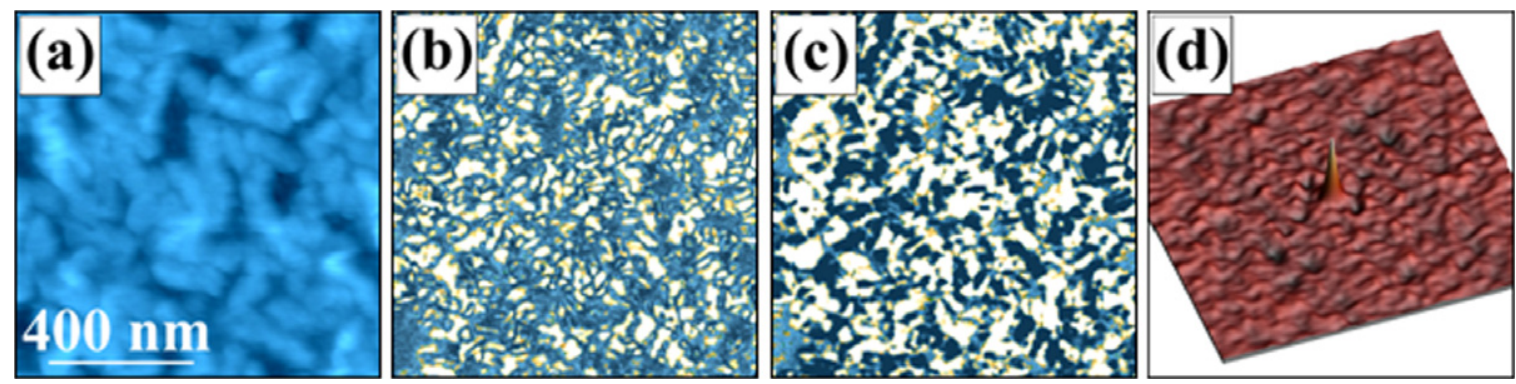

Figure 3. Domain imaging in 12 ML P(VDF-TrFE) film subjected to two cycles of annealing: (a) topography; (b) PFM amplitude; (c) PFM phase; and (d) 3D auto-correlation image of the PFM phase data (scale is arbitrary along the vertical axis).

the chain axis and polarization reversal is associated with rotation of these dipoles about the molecular chain. In the crystalline $\beta$ phase, molecules tend to pack parallel to each other forming a quasi-hexagonal orthorhombic structure with a lattice spacing of approximately $0.5 \mathrm{~nm}$ along the polar axis. In this work, $\mathrm{P}(\mathrm{VDF}-\mathrm{TrFE})$ films with a molar content ratio of 80:20 of various morphological structures and thicknesses were investigated.

Figure 2 shows topographic and PFM images of the $\mathrm{P}(\mathrm{VDF}-\mathrm{TrFE})$ film subjected to one cycle of annealing with a thickness of $12 \mathrm{ML}$ [18]. The topographic image reveals a morphological structure consisting of grains from 200 to $600 \mathrm{~nm}$ in lateral dimensions with rather diffused grain boundaries and average root-mean-square roughness of $7.96 \mathrm{~nm}$ over a $1 \times 1 \mu \mathrm{m}^{2}$ area. The vertical PFM amplitude image of the film (figure 2(b)) generally exhibits a saturated signal indicative of uniform polarization which, given the local switching data, extends through the film thickness from the bottom to the top interface. Given that the polarization vector is nearly perpendicular to the chain axis, a high value of the PFM amplitude signal suggests that the molecular chains in these crystallites are aligned parallel to the substrate, which is the usual case for Langmuir-Blodgett films of this polymer. Domain walls appear as narrow dark lines with a characteristically weak amplitude signal and a smallest measured width of $6 \mathrm{~nm}$.

This value, which is almost an order of magnitude larger than the real physical thickness of the domain wall in ferroelectrics (1-2 unit cells), is a consequence of the finite size of the probing tip and tip-sample contact area. Significant widening of the domain walls in the PFM amplitude observed in some areas might be a result of the presence of amorphous non-polar phase or non-through domains with head-to-head or tail-to-tail configuration. The PFM phase signal (figure $2(\mathrm{c})$ ) changes by $180^{\circ}$ across the domain walls, indicating antiparallel domains with polarization normal to the film surface. Specifically, bright regions correspond to domains with polarization up and dark regions indicate domains with polarization down.

A second annealing of the $12 \mathrm{ML}$ thick sample resulted in a profound change in the film morphology and polarization distribution. Large grains transformed into ricelike elongated crystallites approximately $200 \mathrm{~nm}$ long and $50 \mathrm{~nm}$ wide (figure 3(a)) forming a surface with root-meansquare roughness of $6.25 \mathrm{~nm}$ over a $1 \times 1 \mu \mathrm{m}^{2}$ area. A similar structure has recently been reported by a group from Kyoto University [19, 20]. As can be seen from the PFM images in figures 3(b) and (c), the morphological changes are accompanied by a significant reduction of the average domain size. Voids between the rice-like crystallites could cause additional variations in the PFM amplitude signal in figure 3(b).

To obtain a quantitative description of the polarization distribution, the PFM data have been processed using the image analysis program WSxM v2.2 (Nanotec Electronica) [21]. The average domain size has been estimated by means of autocorrelation analysis. The three-dimensional auto-correlation images (figures 2(d) and 3(d)) have been obtained from the original PFM phase images by using a transformation of the following form:

$$
C\left(r_{1}, r_{2}\right)=\sum_{x, y} D(x, y) * D\left(x+r_{1}, y+r_{2}\right)
$$



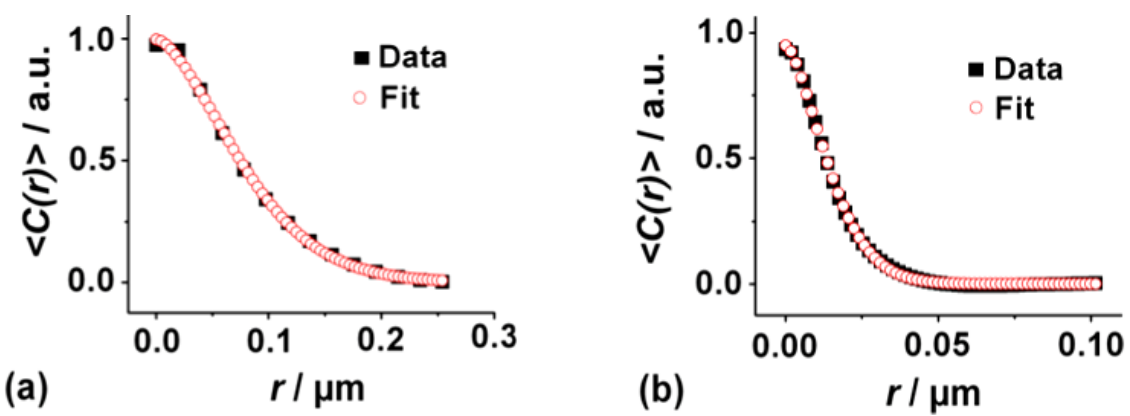

Figure 4. Distance dependence of the auto-correlation function $\langle C(r)\rangle$ averaged over all directions: (a) corresponds to the 3D auto-correlation image in figure 2 and (b) corresponds to the $3 \mathrm{D}$ auto-correlation image in figure 3 . Red open circles represent the best fit to the data points (black squares).
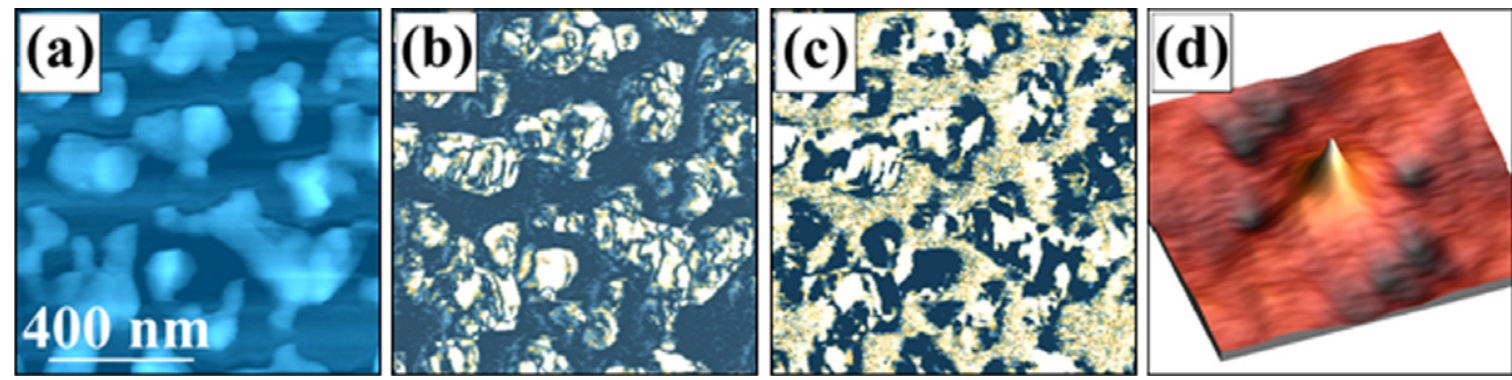

Figure 5. Domain imaging in 3 ML P(VDF-TrFE) film with nanomesa structure: (a) topography; (b) PFM amplitude; (c) PFM phase; and (d) 3D auto-correlation image of the PFM phase data (scale is arbitrary along the vertical axis).
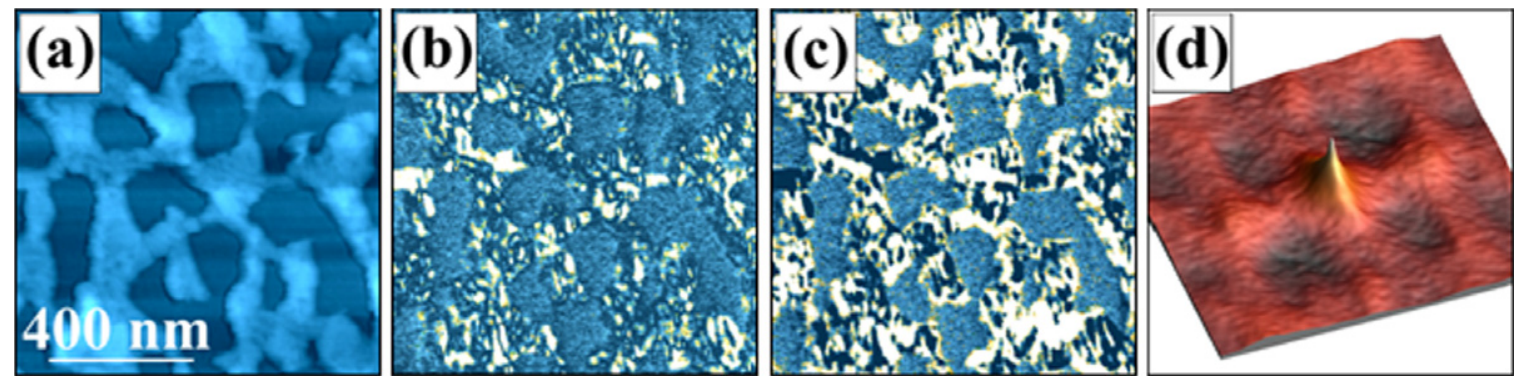

Figure 6. Domain imaging in 3 ML P(VDF-TrFE) film with nanowell morphology: (a) topography; (b) PFM amplitude; (c) PFM phase; and (d) 3D auto-correlation image of the PFM phase data (scale is arbitrary along the vertical axis).

where $D(x, y)$ is the PFM phase signal at a point in the image and $D\left(x+r_{1}, y+r_{2}\right)$ is the PFM phase signal at a point translated by $\left(r_{1}, r_{2}\right)$ from the original reference point. In the auto-correlation image, the peak corresponds to the average size of the areas with correlated polarization, i.e. with the same polarization direction. Only a short range correlation length term was taken into account and the term which accounts for the long range correlation length has been excluded as it is affected by the non-uniform morphology of the films. The behavior of the average auto-correlation function was fitted with the formula [22]

$$
C(r)=A * \exp \left[-(r / \xi)^{2 h}\right]
$$

where $A$ is the normalization constant, $r$ is the radius from the center of the image, $\xi$ is the correlation length and $h$ is a parameter $(0<h<1)$. Equation (2) describes the short range correlation analysis and provides the correlation length $\xi$ averaged over all azimuthal directions.

The best fit yields values of the correlation length $\xi=$ $94 \mathrm{~nm}$ for the $12 \mathrm{ML}$ film annealed once and $\xi=18 \mathrm{~nm}$ for the 12 ML film annealed twice (figure 4) with the exponent value $h=0.8$. The average domain size is approximately twice the correlation length. Hence, the correlation analysis quantifies the decrease in the average domain size as a function of morphological changes.

We continued our studies by analyzing polarization distribution in $3 \mathrm{ML}$ P(VDF-TrFE) films which, depending on annealing conditions, exhibited a drastically different morphology. Specifically, the 3 ML films were not continuous but displayed either regions of irregular nanomesas of 200$500 \mathrm{~nm}$ in size (figure 5(a)) [23] or regions with a meshlike structure, which we refer to as a nanowell structure (figure 6(a)). PFM imaging of these structures revealed 


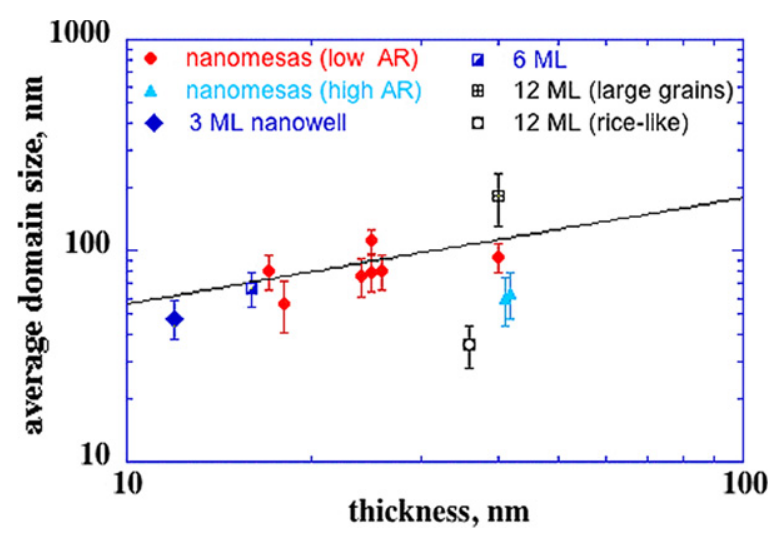

Figure 7. Average domain size versus thickness. Solid line represents Kittel's law behavior.
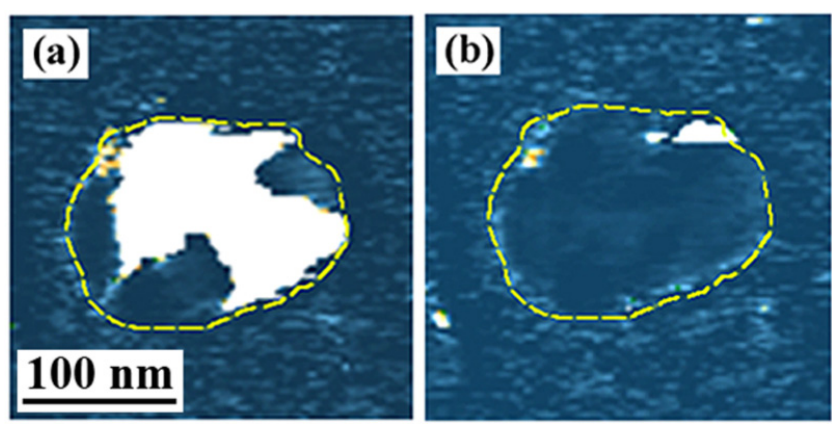

Figure 8. Local switching in a single nanomesa in the $3 \mathrm{ML}$ $\mathrm{P}(\mathrm{VDF}-\mathrm{TrFE})$ film: PFM phase images of a domain structure before (a) and after (b) application of a $-8 \mathrm{~V}, 2 \mathrm{~s}$ voltage pulse.

qualitatively different domain patterns. While relatively large domains were observed in nanomesas (figures 5(b) and (c)), domains in the nanowell structures were much smaller in size (figures 6(b) and (c)). Auto-correlation analysis of the PFM data (figures 5(d) and 6(d)) provided a quantitative insight into the polarization distribution. Namely, the correlation length was found to be $37 \mathrm{~nm}$ in $3 \mathrm{ML}$ nanomesas shown in figure 5 and $12 \mathrm{~nm}$ in $3 \mathrm{ML}$ films with nanowell structure in figure 6 . Domain distribution also strongly depends on film thickness which has been measured using cross-sectional analysis of the topographic images. It has been found that in continuous films and nanomesas with a relatively low thickness/size aspect ratio
$(<1 / 10)$, the average domain size $w$ and film thickness $d$ are related via a well-known Kittel relation, $w=K d^{1 / 2}$, where the constant $K$ describes a balance between the domain wall and electrostatic energies (figure 7) [24, 25]. On the other hand, films with rice-like grains and mesas with high thickness/grain aspect ratio $(>1 / 5)$ exhibit significant deviations from this law.

It is known that, in the absence of the electrodes, domain formation is one of the main mechanisms of minimization of the free energy of a ferroelectric sample. However, for films with high thickness/grain aspect ratio, effects associated with the surface energy can contribute significantly to the formation of thermodynamically stable domain structures. Transformation of $\mathrm{P}(\mathrm{VDF}-\mathrm{TrFE})$ films after annealing into the nanomesas or nanowell structures is governed by a variation of the surface tension at the film-substrate interface. It can be assumed that, in the nanomesas with high aspect ratio, an increase in the surface energy makes formation of additional domain walls energetically unfavorable, thus resulting in larger domains. On the other hand, in continuous films, the surface energy is significantly decreased allowing formation of smaller antiparallel $180^{\circ}$ domains as a means of reducing the overall free energy. Clearly, additional studies on domain structures as a function of annealing conditions are required to clarify the competition between thermodynamically and kinetically driven processes of domain formation.

Testing of the switching behavior in individual mesas has been performed by applying a single voltage pulse through the tip in contact with the mesa surface followed by PFM imaging of the resulting domain pattern. Figure 8 shows representative PFM phase images of a mesa in the 3 ML film of approximately $200 \mathrm{~nm}$ in size. As can be seen, initially the mesa is in a poly-domain state (figure 8(a)). Application of a voltage pulse reverses polarization in the grain, as is illustrated by the change of the PFM contrast (figure 8(b)). Naturally, the size of the switched area depends strongly on the parameters of the switching pulse, with a smallest written domain being about $30 \mathrm{~nm}$ in size. This field-dependent behavior along with the imaging resolution of $6 \mathrm{~nm}$ allows direct studies of domain switching dynamics in nanoscale $\mathrm{P}(\mathrm{VDF}-\mathrm{TrFE})$ mesas.

To get additional insight into the local switching behavior, measurements of the local hysteresis loops have been carried out (figure 9) in the remanent (field-off) state. It has been found that the films do not show any imprint behavior, i.e. the switching is quite symmetric, as there is no disparity between
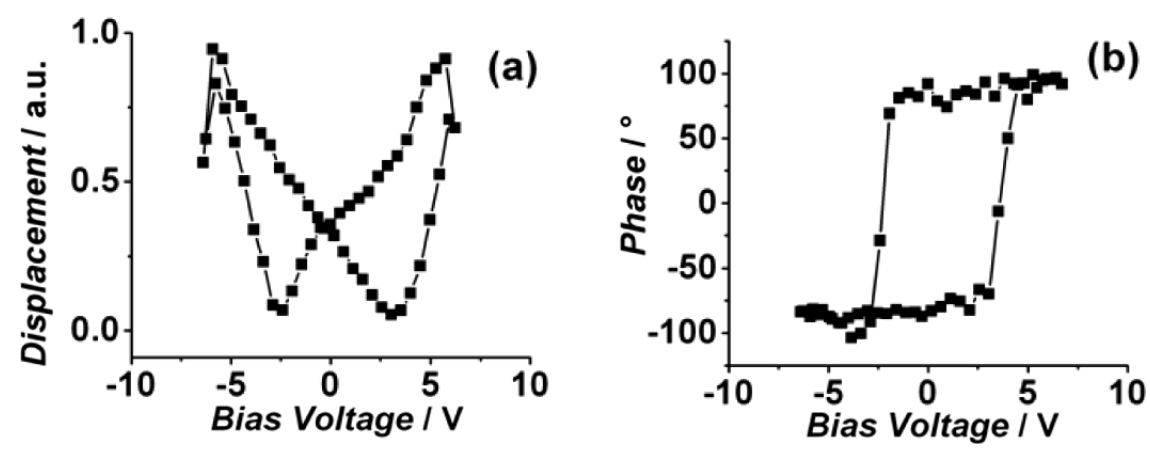

Figure 9. Local hysteresis loops measured in the P(VDF-TrFE) nanomesas: (a) PFM amplitude; and (b) PFM phase. 
positive and negative coercive voltages. The fact that the loop does not saturate can be attributed to an increased electrostatic contribution at a high bias. Further studies of local switching behavior are underway in our group.

\section{Conclusion}

In summary, high-resolution PFM studies of ultrathin polymer $\mathrm{P}(\mathrm{VDF}-\mathrm{TrFE})$ films showed significant variations of domain patterns with changes in film morphology and thickness. It has been found that, for continuous films and nanomesas with relatively low thickness/grain aspect ratio $(<1 / 10)$, the relationship between the average domain size and thickness follows the Kittel law, while films with rice-like grains and nanomesas with high aspect ratio $(>1 / 5)$ exhibit significant deviations from this law, suggesting additional surface-energyrelated mechanisms affecting domain patterns. Polarization reversal within a single crystallite has been demonstrated and local switching parameters (coercive voltage and remnant piezoresponse) have been measured by monitoring the local hysteresis loops. Reliable control of polarization at the sub-grain level demonstrates a possibility of studying the mechanism of the intrinsic switching behavior down to the molecular scale.

\section{Acknowledgments}

The authors would like to express their gratitude to Dr J Y Li, Washington State University, for useful discussions and to Dr V Shvartsman, University of Duisburg-Essen, for valuable advice. This work was supported by the National Science Foundation (grant nos. MRSEC DMR-0820521, ECS-0600130 and NIRT DMR-0403871) and the Nebraska Center for Materials and Nanoscience at the University of Nebraska Lincoln. VMF thanks the Russian Foundation for Basic Research (grants 05-0216871 and 09-02-00096) for their support.

\section{References}

[1] Bharti V, Zhao X-Z, Zhang Q M, Romotowski T, Tito F and Ting R 1998 Mater. Res. Innovat. 257
[2] Lovinger A 1983 Science 2201115

[3] Kimura K and Ohigashi H 1986 Japan. J. Appl. Phys. 25383

[4] Ducharme S, Fridkin V M, Bune A V, Palto S P, Blinov L M, Petukhova N N and Yudin S G 2000 Phys. Rev. Lett. 84175

[5] Hu Z, Tian M, Nysten B and Jonas A M 2009 Nat. Mater. 862

[6] Furukawa T 1989 Phase Transit. 18143

[7] Güthner P and Dransfeld K 1992 Appl. Phys. Lett. 611137

[8] Matsushige K, Yamada H, Tanaka H, Horiuchi T and Chen X Q 1998 Nanotechnology 9208

[9] Rodriguez B J, Jesse S, Kim J, Ducharme S and Kalinin S V 2008 Appl. Phys. Lett. 92232903

[10] Bystrov V S, Bdikin I K, Kiselev D A, Yudin S, Fridkin V M and Kholkin A L 2007 J. Phys. D: Appl. Phys. 404571

[11] Gysel R, Stolichnov I, Tagantsev A K, Setter N and Mokr P 2008 J. Appl. Phys. 103084120

[12] Rodriguez B J, Jesse S, Kalinin S V, Kim J, Ducharme S and Fridkin V M 2007 Appl. Phys. Lett. 90122904

[13] Ducharme S, Palto S P and Fridkin V M 2002 Handbook of Thin Film Materials vol 3, ed H S Nalwa (San Diego, CA: Academic)

[14] Sorokin A, Palto S, Blinov L, Fridkin V M and Yudin S 1996 Mol. Mater. 661

[15] Bai M, Poulsen M and Ducharme S 2006 J. Phys.: Condens. Matter 187383

[16] Li J, Luo Y, Bai M and Ducharme S 2005 Appl. Phys. Lett. 87213116

[17] Gruverman A, Wu D and Scott J F 2008 Phys. Rev. Lett. 100097601

[18] Bai M, Sorokin A V, Thompson D W, Poulsen M, Ducharme S, Herzinger C M, Palto S, Fridkin V M, Yudin S G, Savchenko V E and Gribova L K 2004 J. Appl. Phys. 953372

[19] Kimura K, Kobayashi K, Yamada H, Horiuchi T, Ishida K and Matsushige K 2004 Japan. J. Appl. Phys. 434575

[20] Fukuma T, Kobayashi K, Horiuchi T, Yamada H and Matsushige K 2000 Japan. J. Appl. Phys. 393830

[21] Horcas I, Fernandez R, Gomez-Rodriguez J M, Colchero J, Gomez-Herrero J and Baro A M 2007 Rev. Sci. Instrum. 78013705

[22] Shvartsman V V and Kholkin A L 2007 J. Appl. Phys. 101064108

[23] Bai M and Ducharme S 2004 Appl. Phys. Lett. 853528

[24] Kittel C 1946 Phys. Rev. 70965

[25] Mitsui T and Furuichi J 1953 Phys. Rev. 90193 